\title{
Oral Microbiome and Health
}

\author{
Ketevan Nanobashvili ${ }^{1 *}$, Lia Mania ${ }^{2}$ and Rusudan Ivanishvili ${ }^{3}$ \\ ${ }^{1}$ Professor, Department of Dentistry, University of Georgia, Tbilisi, Georgia \\ ${ }^{2}$ Assistant Professor, Faculty of Public Health, Dental Program, Tbilisi Humanitarian \\ Teaching University, Tbilisi, Georgia \\ ${ }^{3}$ Associate Professor, Department of Dentistry, University of Georgia, Tbilisi, Georgia \\ *Corresponding Author: Ketevan Nanobashvili, Professor, Department of \\ Dentistry, University of Georgia, Tbilisi, Georgia.
}

Received: May 26, 2021

Published: June 29, 2021

(C) All rights are reserved by Ketevan

Nanobashvili., et al.

\section{Abstract}

The human oral cavity is one of the most diverse environments in terms of microbial settlement. The microbiotic environment of the oral cavity includes viruses, fungi, protozoa, archaea, and bacteria. From the listed microorganisms bacterial microflora are present in the largest numbers, namely - Phyla Actinobacteria, Bacteroidetes, Firmicutes, Proteobacteria, Spirochaetes, Synergistetes and Tenericutes [1].

Oral resident microorganisms and their interactions are an essential component of changing the balance between health and disease. The main subject of studying microbiology is to understand the role of the microbial community in the oral cavity, gastrointestinal tract and respiratory tract in terms of health or disease [3].

Based on numerous studies, we come to the conclusion that the oral microflora is still different for each individual and may have been influenced by factors such as: time [10], age [11], nutritional peculiarities [12], extreme environment [13], stress [14].

Definitely worth mentioning that the oral microbiome can have a significant impact on the development of the oral or systemic pathology, and it is related to the imbalance of the microflora [16].

Microbial dysbiosis of the oral cavity is associated with inflammatory diseases of the oral cavity and may contribute to the aggravation of the systemic conditions by bacteremia. The oral cavity as a concept a source of distant infection has been considered for at least a century [26].

Consequently, dysbiosis of the oral microbiome can be associated with diseases of several organ systems such as the gastrointestinal tract [10,27], cardiovascular system [28]. Damage to the endocrine and nervous systems [10], to the immune system [29]. The connection of each system with the change of the microbial society of the oral cavity, prior to the clinical manifestation of the disease, the quantitative abundance of certain species and the potential for increased virulence approved by reliable studies.

Given the current epidemiological situation in the world, determines our interest to changes in the oral cavity and in their biomass composition of individuals infected with SARS-CoV-2. Several recent publications have been reviewed for this purpose. It is possible to assume that by studying the oral microbiome of those infected with Covid-19 will be improved the safety of guidelines of modern dental manipulations, improve infection control capabilities in dental practice, and make the delivery of dental services more secure. It would be even more interesting to study the prevalence of oral diseases such as caries and periodontal disease in new infection conditions, oral mucosal diseases in all age groups, changes in their oral biome over time and subsequent impact on general health.

Citation: Ketevan Nanobashvili., et al. "Oral Microbiome and Health". Acta Scientific Dental Sciences 5.7 (2021): 138-144. 
It is noteworthy that timely study of secondary attachment infections in co-infected patients is required. The urgency of the issue is clear, as knowledge about the expected oral manifestations of SARS-CoV-2- in the mouth of individuals infected with the infection is still scarce. The issue needs further study. We can boldly hypothesize that oral microbiome will be altered in patients infected with Covid and that an in-depth study of this issue would be interesting. It is desirable to emphasize the need for close cooperation between dentists and infectious disease specialists. As well as the prevention of opportunistic infections of the oral cavity in patients infected with Covid-19 through complete oral hygiene and enhancement of local immune forces. This in itself implies the rehabilitation of the oral cavity and the strengthening of preventive measures against oral diseases in the first line of public health in both healthy and co-infected patients.

Keywords: Oral Microbiome; Oral Diseases; Microflora and Factors; Oral Manifestations of Sar-Covid-19-Infection

\section{Introduction}

The human oral cavity is one of the most diverse environments in terms of microbial settlement. The microbiotic environment of the oral cavity includes viruses, fungi, protozoa, archaea, and bacteria. From the listed microorganisms bacterial microflora are present in the largest numbers, namely - Phyla Actinobacteria, Bacteroidetes, Firmicutes, Proteobacteria, Spirochaetes, Synergistetes and Tenericutes [1].

The oral cavity, after the gastrointestinal tract, is in second place in terms of microbial diversity. According to HOMD (last update 2017), 772 prokaryotic microbes are observed in the oral cavity, of which $70 \%$ are cultivable and $30 \%$ belong to the unprocessed class of microorganisms.

Of the $70 \%$ of cultured species of healthy oral microflora, $57 \%$ have been classified as 16SrDNA profiling into 6 main types: Firmicutes, Actinobacteria, Proteobacteria, Fusobacteria, Bacteroidetes and Spirochaetes. They directly affect human health - from the metabolism of the host organism, ending with - immune reactions. Changes in the oral microflora have been observed in several diseases such as diabetes, bacteremia, endocarditis, cancer, autoimmune disease and premature birth. Through this information and microbiological research methods, it is possible to create microbiome-based biomarkers, They are used in the early diagnosis of oral cavity and related diseases [2].

Oral resident microorganisms and their interactions are an essential component of changing the balance between health and disease. The main subject of studying microbiology is to understand the role of the microbial community in the oral cavity, gastrointestinal tract and respiratory tract in terms of health or disease [3].
Currently, it is accepted by the medical community that under normal conditions, prenatal development of the fetus occurs in an aseptic environment. During and after birth, the newborn comes into contact with numerous microorganisms. An important part of the first colonizing bacteria in the human body is of maternal origin. The type of birth - autochthonous or dystocian, can affect the type of microorganisms that the newborn is firstly exposed to. In 24 hours after birth in the oral cavity of the newborn, the presence of the so-called pioneer microorganisms is noted. At this time, the oral cavity is most often colonized by gram-positive cocci, among which Streptococcus and Staphylococcus are particularly noteworthy [4].

At about five months of age, infants already have a different oral microbiota from their mothers. It is formed under the influence of various factors: environmental impact in the first months of life, food intake, contact with other adults and children, contact with pets, hygiene, habits, etc. This microbiota consists mainly of six bacterial phyla: Proteobacteria, Actinobacteria, Bacteroidetes, Fusobacteria and Spirochaetes. The main common genera among them are: Streptococcus, Haemophilus, Neisseria and Veillonella [5].

At the three years old of age saliva microbiome is already complicated, however, the process of its maturation lasts until adulthood. In childhood, the oral microbiota changes throughout the period of tooth development. During periods of primary, mixed or permanent occlusions. Compared to other age groups, the oral microbiota of children with primary occlusion is characterized by a higher composition of bacteria of the class Gammaproteobacteria, especially with samples of family Pseudomonaceae (genus Pseudomonas); Also - Moraxellaceae (genera Acinetobacter, Moraxella and Enhydrobacter), Enterobacteriaceae and Pasteurellaceae (Aggregatibacter genus) [6]. 
In contrast to adults, the oral microflora of a healthy child is mostly represented by bacterial flora - phyla Firmicutes (genus Streptococcus, Veillonella, Lactobacillus and Granulicatella) and Actinobacteria (genera Rothia and Actinomyces); Marked in small quantities representatives of phyla Bacteroidetes (genus Prevotella, order Bacteroidales), also - Fusobacteriae (Fusobacterium genus), Spirochaetes and in small quantity - TM7 (Saccharibacteria).

The amount of periopathogenic bacteria increases with the age of the child. A change in the bacterial population from aerobic or facultative gram-positive cocci to anaerobic to fastidious gramnegative bacteria has been observed [7].

Puberty is a period of hormonal changes, it is accompanied by significant changes in the environment of the oral cavity, and it is more related to the diversity of food. This phenomenon leads to the growth of a group of some microorganisms in the oral cavity, among which gram-negative anaerobes and spirochetes are particularly noteworthy [8]. This change in the oral microbiota may be associated with increased incidence and severity of gingivitis during puberty [9].

Based on numerous studies, we come to the conclusion that the oral microflora is still different for each individual and may have been influenced by factors such as:

- Time: A 12 - 18 month study by the HMP Consortium, which examined the structure and function of microbiome in 18 areas of the body of 300 healthy adults, found that the oral cavity was the most unstable in this regard [10].

- Age: Also interesting is the study of oral microbial flora conducted in healthy and well-controlled (managed) HIVinfected children living in the USA. The studies found that the microbial settlement of the oral cavity in both groups of children were virtually the same. However, differences were observed in the microbiome of children with permanent and primary dentition. Differences associated with age have been reported in species such as Prevotella intermedia, Porphyromonas gingivalis and $P$. Nigrescens. The percentage of those children, in which the target species could not be identified, was $40 \%$ at the age of 3 years old and $0 \%$ at the age of -11. It even underlines strict dependence on age for oral microbiome changes [11].
- Nutrition feature: For the research the researchers took saliva samples from three pairs of hunter-gatherers and traditional farmers living near the Philippines. The study found that significant dietary changes chosen for various commensals played a role in the development of modern oral pathogens [12].

- Extreme environment: The study monitored changes in microbial populations taken from different parts of the mouth before and after flight [13].

- Stress: One of the factors that cause microbiome dysbiosis has been found to be physiological stressors. Evolutionarily, human microorganisms have the ability to perceive signals associated with hormonal changes in the host body. In order to adapt to a new environment, they respond in the form of changes in their own genetic information. Studies have shown the effects of the stress hormone cortisol on the oral microbiome, which in turn contributes to the development and progression of periodontal disease [14].

- Other factors: HMP studies have also shown a strong association between the microcosm in different parts of the body and factors such as breastfeeding, gender, level of education, etc [15].

Oral microbiota and oral diseases

It should be noted that the oral microbiome can have a significant impact on the development of oral and systemic pathology, which is related to the imbalance of the microflora. The use of current molecular methods has significantly increased awareness of the composition and function of the oral microbiome in health and disease. The study of oral microbiomes under different health conditions and their interactions with microbiomes in different parts of the body play an important role in understanding our body and improving general health [16].

\section{Caries}

For study purpose was used the human oral microbe identification microarray (HOMIM) to compare bacterial profiles of saliva and supragingival plaque in children with SECC and children without caries. Approximately 379 bacterial species and several genera were found in all samples. Among them, Streptococcus, Porphyromonas and Actinomyces have been found to be particularly associated with the SECC. At the same time, these microorganisms 
can be considered as potential biomarkers of dental caries during the period of primary dentition [17].

By other researchers have compared the saliva of children suffering with dental caries and caries-free children by single-molecule DNA Sequencing analysis. It has been established that Prevotella spp., Lactobacillus spp., Dialister spp and Filifactor spp. are associated with the development of dental caries and if they are detected, it is possible to predict the onset of caries [18].

Other authors used the NGS to analyze the microbiome of the first Canadian resident and metis children with- and without SECC. Very high levels of Streptococcus mutans were observed in the SECC group [19].

Periodontal disease develops when the number of gram-negative bacteria and anaerobes in the subgingival plaque increases. Numerous studies have been conducted to identify the types of bacteria associated with periodontal disease. Studies have shown that the most important species associated with periodontal disease are microorganisms such as Aggregatibacter (Actinobacillus), Porphyromonas gingivalis, Tannerella forsythensis, and spirochaete, Treponema denticola. Modern studies have considered the pathogenesis of periodontitis in immunocompromised children includeb microorganisms such as fungi - Candida albicans and herpesviruses [20].

An interesting case-control study was conducted to identify the possible association of juvenile periodontitis (JP) with pathogenic bacteria and viruses such as Cytomegalovirus (HCMV) and the Epstein-Barr virus type 1 (EBV-1).

The study was conducted among school aged children from north-central Jamaica. Samples from subgingival plaque were taken based on the following data: out of 15 subjects - diagnosed with JP, from 20 subjects - diagnosis incipient periodontitis (IP) And 65 from the representatives of a randomly selected healthy control group. 16S rRNA polymerase chain reaction (PCR) study revealed the presence of microorganisms such as Porphyromonas gingivalis and Actinobacillus actinomycetemcomitans and HCMV and EBV-1 using the PCR identification method.

Interestingly, the study samples, which simultaneously showed the presence of $P$ gingivalis and HCMV, were associated with significant variables of clinical attachment loss in the case of juvenile periodontitis [21].
Diseases of the oral mucosa

Diseases of the oral mucosa such as oral leukoplakia (OLK), oral lichen planus (OLP), and systemic lupus erythematosus (SLE) are common independent diseases of the oral mucosa or a specific manifestation of systemic diseases on the oral mucosa. Several studies have shown that bacteria play an important role in the initiation and progression of these mucosal diseases [22].

Also noteworthy are oncological diseases of the oral mucosa, the origin and development of which are influenced by several factors: hereditarity, microflora (bacteria), general condition of the body, etc. The existence of a link between microbiome and oral cancer has been proven by numerous studies $[10,16]$.

A study was conducted in which saliva from 45 patients with OSSC was compared to 229 healthy individuals from control group. The study found that in patients with OSSC, compared with the control group, there was a significant excess of 6 species of microorganisms: P. melaninogenica, Leptotrichia buccalis, Capnocytophaga ochracea, C. gingivalis, Eubacterium saburreum and S. mitis. In case of the presence of three species of microorganisms in the microbiome (C. gingivalis, P. melaninogenica and S. mitis), they can be used as diagnostic markers of oral cancer with $80 \%$ of confidence [23].

The relationship between changes in the oral microflora of cancer patients and chemotherapy for therapeutic purposes has been studied [24]. Data were also examined to confirm a possible link between candida and oral cancer. Researchers have determined the causal role of candidiasis in the development of precancerous diseases and cancer of the oral cavity; It has also been shown that the presence of Candida, along with other co-factors, influences the onset and development of carcinogenesis. The authors suggest that the nitrosation potential of $C$. albicans leads to the production of carcinogenic nitrosamine, which makes the oral epithelium prone to dysplastic changes, which in turn is a contributing factor to the development of carcinoma. Subsequently, under the influence of other factors such as disruption of the oral mucosa and tobacco smoking, the virulence of the microorganism may be enhanced [25].

\section{Oral microbiota and systemic diseases}

The oral cavity is the introductory part of digestive and respiratory tract. More than 700 bacterial species can be found in the human oral cavity [26]. Microbial dysbiosis of the oral cavity is 
associated with inflammatory diseases of the oral cavity and may contribute to the aggravation of the systemic condition by bacteremia. The concept of the oral cavity as a source of distant infection, it has been considered for a century [27].

Consequently, dysbiosis of the oral microbiome can be associated with diseases of several organ systems such as the gastrointestinal tract $[10,28]$, cardiovascular system [29]. Damage to the endocrine and nervous systems [10], to the immune system [30]. The connection of each system with the change of the microbial society of the oral cavity, prior to the clinical manifestation of the disease, the quantitative abundance of certain species and the potential for increased virulence approved by reliable studies.

Our attention was especially payed to the changes in the oral microbiome during the immune system functional changes and infectious diseases. Infectious diseases of pediatric age, such as measles, rubella, chicken-pox, and herpes virus infections, show only an increase in the virulence of the local microflora and a high risk of opportunistic infection [31-33].

It should be noted that quite significant changes are observed in the examination of AIDS patients. In particular: Firmicutes and Streptococcus sp. are found in the supragingival biofilm of HIVinfected subjects compared with non-infected individuals (95\% and $78 \%$ confidence). In the subgingival biofilm of the HIV-infected subjects, relative abundance of genera Veillonella sp. and the Prevotella $s p$. was higher than in non-infected individuals. Neisseria $s p$. was found in relatively large numbers on the tongue of HIV-infected individuals (21\%). In saliva genera Prevotella sp. difference between non-infected and HIV-infected individuals was $15 \%$ and $7 \%$, respectively. A prevalence of both - subgingival and supragingival biofilms in HIV-1-infected individuals were dected by Chao-index compared with non-infected individuals [34].

Given the current epidemiological situation in the world, determines our interest to changes in the oral cavity and in their biomass composition of individuals infected with SARS-CoV-2. Several recent publications have been reviewed for this purpose.

Unfortunately, knowledge about the expected oral manifestations is still scarce, this may be due to the frequent remote control of patients infected with Covid-19 and their inability to undergo intraoral inspection; This is due to the existing regulations and restrictions. However, several cases of oral manifestations are found in the literature [35].
In the case-reports, the authors indicated that patients complained of pain in the facial area, on the tongue; The presence of ulcerative and vesicular lesions in the oral cavity; Patients had lesions on attached mucosa that mimics herpes simplex. But they did not have a history of herpes infection. The oral manifestations of some infected individuals were very similar to multiform exudative erythema. Because patients were consulted remotely and using telemedicine methods, unfortunately, the researchers did not have the opportunity to provide a biopsy, or other type of laboratory tests, which required physical contact with the patient. Accordingly, they make recommendations for further studies to determine whether these lesions are caused by a new infection and may be considered a clinical sign of it or these changes are developed as the result of emotional stress [36].

Bacterial coinfections are often the cause of complications and increased mortality in people with respiratory viral infections. Therefore, studies have been conducted, where researchers hypothesized that in confirmed increased cases of Covid-19 would spontaneously increase complication of acute periodontal disease, particularly necrotic periodontal disease (NPD), which would be associated with bacterial co-infections. A metagenomic analysis was performed in covid-infection patients with acute respiratory syndrome, which confirmed the presence of a very large number of bacteria Prevotella intermedia - In addition, major pathogens such as Streptococci, Fusobacterium, Treponema and Veillonella were involved in co-infection during disease onset and progression [37]. In general, periodontal necrotic disease (NPD) is more common in HIV-infected patients. It is possible, that periodondal necrotic diseases may be developed by the same mechanism in patients infected with covid-virus, which is provoked by the presence of Prevotella intermedia. It is important to urgently investigate secondary co-infections in covid-patients.

\section{Conclusion}

It is possible to assume that by studying the oral microbiome of those infected with Covid-19 will be improved the safety of guidelines of modern dental manipulations, improve infection control capabilities in dental practice, and make the delivery of dental services more secure.

It would be even more interesting to study the prevalence of oral diseases such as caries and periodontal disease in new infection conditions, oral mucosal diseases in all age groups, changes 
in their oral biome over time and subsequent impact on general health.

It is noteworthy that timely study of secondary attachment infections in co-infected patients is required. The urgency of the issue is clear, as knowledge about the expected oral manifestations of SARS-CoV-2-in the mouth of individuals infected with the infection is still scarce. The issue needs further study. We can boldly hypothesize that oral microbiome will be altered in patients infected with Covid and that an in-depth study of this issue would be interesting. It is desirable to emphasize the need for close cooperation between dentists and infectious disease specialists. As well as the prevention of opportunistic infections of the oral cavity in patients infected with Covid-19 through complete oral hygiene and enhancement of local immune forces. This in itself implies the rehabilitation of the oral cavity and the strengthening of preventive measures against oral diseases in the first line of public health in both healthy and co-infected patients.

\section{Bibliography}

1. William G Wade. "The oral microbiome in health and disease". Pharmacological Research 69.1 (2013): 137-143.

2. Digvijay Verma., et al. "Insights into the human oral microbiome”. Archives of Microbiology 200 (2018): 525-540.

3. Benedita Sampaio-Maia and Filipa Monteiro-Silva. "Acquisition and maturation of oral microbiome throughout childhood: An update". Dental Research Journal 11.3 (2014): 291-301.

4. Bagg J., et al. Essentials of Microbiology for Dental Students. New York: Oxford University Press (2006).

5. Cephas KD., et al. "Comparative analysis of salivary bacterial microbiome diversity in edentulous infants and their mothers or primary caregivers using pyrosequencing". PloS one 6 (2011): e23503.

6. Crielaard W., et al. "Exploring the oral microbiota of children at various developmental stages of their dentition in the relation to their oral health". BMC Medical Genomics 4 (2011): 22.

7. Tanner AC., et al. "Similarity of the oral microbiota of preschool children with that of their caregivers in a populationbased study". Oral Microbiology and Immunology 17 (2003): 379-387.
8. Lamont R., et al. "Oral Microbial Ecology”. In: Lamont R, Hajisjengallis GN and Jenkinson $\mathrm{H}$. "Oral microbiology and immunology. Washington DC: ASM Press (2014): 89-105.

9. Mombelli A., et al. "Gingival health and gingivitis development during puberty. A 4-year longitudinal study". Journal of Clinical Periodontology 16 (1989): 451-456.

10. Gao L., et al. "Oral microbiomes: more and more importance in oral cavity and whole body". Protein Cell 9 (2018): 488-500.

11. Goldberg Brittany E., et al. "The Oral Bacterial Communities of Children with Well-Controlled HIV Infection and without HIV Infection (2015).

12. Lassalle F., et al. "Oral microbiomes from hunter-gatherers and traditional farmers reveal shifts in commensal balance and pathogen load linked to diet". Molecular Ecology 27.1 (2017): 182-195.

13. Brown LR., et al. "Effect of Skylab missions on clinical and microbiologic aspects of oral health". Journal of the American Dental Association 93.2 (1976): 357-363.

14. Duran-Pinedo AE., et al. "The effect of the stress hormone cortisol on the metatranscriptome of the oral microbiome". NPJ Biofilms Microbiomes 4 (2018): 25.

15. Anukam KC and Agbakoba NR. "A comparative study of the oral microbiome compositions of healthy postmenopausal, premenopausal, and prepubertal Nigerian females, using $16 \mathrm{~s}$ rrna metagenomics methods". Nigerian Journal of Clinical Practice 20.10 (2017): 1250.

16. Vijayan Srinivasprasad., et al. "Liaison between micro-organisms and oral cancer". Journal of Pharmacy And Bioallied Sciences 7.2 (2015): S354-S360.

17. Ma C., et al. "Comparison of oral microbial profiles between children with severe early childhood caries and caries-free children using the human oral microbe identification microarray". PLoS ONE 10.3 (2015): e0122075.

18. Wang Y., et al. "Profiling of oral microbiota in early childhood caries using single-molecule real-time sequencing". Frontiers in Microbiology 8 (2017): 2244.

19. Agnello M., et al. "Microbiome associated with severe caries in Canadian First Nations Children". Journal of Dental Research 96.12 (2017): 1378-1385. 
20. Al-Ghutaimel Hayat., et al. "Common Periodontal Diseases of Children and Adolescents". International Journal of Dentistry (2014): 850674.

21. Michalowich Bryan S., et al. "Human Herpesviruses and Porphyromonas gingivalis Are Associated With Juvenile Periodontitis". Journal Per 71.6 (2000): 981-988.

22. Hu X., et al. "Changes in the salivary microbiota of oral leukoplakia and oral cancer". Oral Oncology 56 (2016): 6-8.

23. Sixou JL., et al. "Modifications of the microflora of the oral cavity arising during immunosuppressive chemotherapy". European Journal of Cancer Part B: Oral Oncology 32B (1996): 306310.

24. Napeñas JJ., et al. "Relationship between mucositis and changes in oral microflora during cancer chemotherapy". Oral Surgery, Oral Medicine, Oral Pathology, Oral Radiology, and Endodontology 103 (2007): 48-59.

25. Sanjaya PR., et al. "Candida in oral pre-cancer and oral cancer". Medical Hypotheses 77 (2011): 1125-1128.

26. Paster BJ., et al. "The breadth of bacterial diversity in the human periodontal pocket and other oral sites". Periodontology 200042 (2006): 80-87.

27. Han YW and Wang X. "Mobile microbiome: oral bacteria in extra-oral infections and inflammation". Journal of Dental Research 92 (2013): 485-491.

28. Michael JD., et al. "Influence of nutrition, body condition, and metabolic status on reproduction in female beef cattle: A review". Theriogenology 125 (2019): 277-284.

29. Thangam Menon., et al. "Characterisation of the human oral microbiome in patients with coronary artery disease using next-generation sequencing of 16SrRNA amplicons". Indian Journal of Medical Microbiology 35 (2017): 101-104.

30. Zheng H., et al. "Subgingival microbiome in patients with healthy and ailing dental implants". Evidence-Based Dentistry 5 (2015): 10948.

31. Mayers Sandra and Curran Alice E. "General and Oral Pathology for Dental Hygienist Practice". Springer (2014): 78-88.

32. Misin A., et al. "Measles: An Overview of a Re-Emerging Disease in Children and Immunocompromised Patients". Microorganisms 8 (2020): 276.
33. Kolenchukova OA., et al. "Staphylococcal colonization of the oral cavity of children following an acute form of rubella infection". Zhurnal Mikrobiologii, Epidemiologii i Immunobiologii 3 (2002): 48-51.

34. Li Y., et al. "HIV Infection and Microbial Diversity in Saliva". Journal of Clinical Microbiology 52.5 (2014): 1400 -1411.

35. Ciccarese G., et al. "Oral erosions and petechiae during SARSCoV-2infection”. Medicine 93 (2021): 129-132.

36. Sinadinos A and Shelswell J. "Oral ulceration and blistering in patients with COVID-19". Evidence-Based Dentistry 21 (2020): 49.

37. Patel Jay and Woolley Julian. "Necrotizing periodontal disease: Oral manifestation of COVID-19". Oral Diseases (2020): 1-2.

\section{Volume 5 Issue 7 July 2021}

(C) All rights are reserved by Ketevan Nanobashvili., et al. 